\title{
Darkogenesis: A baryon asymmetry from the dark matter sector
}

\author{
Jessie Shelton $^{1, *}$ and Kathryn M. Zurek ${ }^{2, \dagger}$ \\ ${ }^{1}$ Department of Physics, Yale University, New Haven, Connecticut 06520, USA \\ ${ }^{2}$ Michigan Center for Theoretical Physics, Department of Physics, University of Michigan, Ann Arbor, Michigan 48109, USA
}

(Received 24 August 2010; published 9 December 2010)

\begin{abstract}
In standard models of baryogenesis and of dark matter, the mechanisms which generate the densities in both sectors are unrelated to each other. In this paper we explore models which generate the baryon asymmetry through the dark matter sector, simultaneously relating the baryon asymmetry to the dark matter density. In the class of models we explore, a dark matter asymmetry is generated in the hidden sector through a first-order phase transition. Within the hidden sector, it is easy to achieve a sufficiently strong first-order phase transition and large enough $C P$ violation to generate the observed asymmetry. This can happen above or below the electroweak phase transition, but in both cases significantly before the dark matter becomes nonrelativistic. We study examples where the asymmetric dark matter density is then transferred to the baryons both through perturbative and nonperturbative communication mechanisms, and show that in both cases cosmological constraints are satisfied while a sufficient baryon asymmetry can be generated.
\end{abstract}

\section{HIDDEN SECTOR BARYOGENESIS}

The standard model (SM) of particle physics has proven remarkably successful at describing the phenomena observed at colliders, from the detailed properties of the gauge sector to flavor physics at bottom and charm factories. Despite this unprecedented success, we know that the SM must be incomplete. Two fundamental features of the observed Universe cannot be explained within the SM: the presence of the baryon asymmetry and the existence of dark matter (DM). The SM contains neither sufficient $C P$ violation to produce the observed size of the baryon asymmetry, nor a particle which can act as DM.

Typically the solutions to these two puzzles are treated independently. Observationally it is known that the DM and baryon densities are approximately the same,

$$
\frac{\rho_{\mathrm{DM}}}{\rho_{b}} \approx 5
$$

However, in most models the DM and baryon densities are not directly related to each other. For example, within the minimal supersymmetric standard model (MSSM), the near-equality of dark and visible relic densities can be accommodated as a coincidence, since $\mathcal{O}(1) C P$ violating parameters together with a $\mathrm{TeV}$ mass scale can give rise to both a thermal relic abundance of a weakly interacting DM particle and an asymmetric relic abundance of baryonic matter, satisfying Eq. (1) (see e.g. [1,2]). However, within the MSSM, it is just as natural for the DM and baryon densities separately to be several orders of magnitude different than their observed values, so that the MSSM does not explain why dark and visible densities appear to be so closely related to each other.

\footnotetext{
*j.shelton@yale.edu

†kzurek@umich.edu
}

An alternate approach is to take the DM relic density to be asymmetric, set by the asymmetry between DM and anti-DM, $n_{X}-n_{\bar{X}}$, just as the baryonic relic density. Relating the DM number asymmetry to baryon number then provides a solution to the puzzle of why the DM and baryon energy densities are so close to each other. Models of this type [3-5] (called asymmetric dark matter [ADM] by [5]) have sharply different phenomenology than thermal models; in particular, the natural scale for ADM is several $\mathrm{GeV}$, since

$$
m_{\mathrm{DM}}=c \frac{\rho_{\mathrm{DM}}}{\rho_{b}} m_{p}
$$

where $c$ is an $\mathcal{O}(1)$ number whose exact size is set by the details of the transfer mechanism. However, much heavier ADM can be possible if there is a coincidence of scales, such that the DM is becoming nonrelativistic just as the operator relating dark number to baryon number is decoupling, as in [6,7], or if there is a cancellation between the injected baryon, lepton, and dark number asymmetries, as in [8].

Tying baryon number to DM number neatly explains the coincidence problem, but does not in itself address the origin of the asymmetry, only its distribution between sectors. In this paper we develop models of ADM where the dark sector itself is responsible for generating the observed matter-antimatter asymmetry.

In order to generate a nonzero baryon asymmetry, the Sakharov conditions must be satisfied [9]:

(i) baryon or lepton number violation,

(ii) departure from thermal equilibrium, and

(iii) $C$ and $C P$ violation.

When the dark sector is responsible for originating the asymmetry, these conditions become: 
(i) the hidden sector must furnish a departure from thermal equilibrium;

(ii) the hidden sector global symmetry stabilizing the DM as well as either baryon $(B)$ or lepton $(L)$ number must be broken by one or more hidden sector processes, while the couplings between the dark sector and the SM must conserve a linear combination of the dark global symmetry, $B$, and/ or $L$; and

(iii) both $C$ and $C P$ must be violated in the dark sector.

We choose to focus on the scenario where the departure from equilibrium is provided by a first-order phase transition in the dark sector, in which the SM does not directly participate. The observed matter-antimatter asymmetry is then entirely generated at this phase transition. We describe the minimal hidden sector which is capable of meeting the Sakharov criteria for generating the asymmetry in the hidden sector. After the asymmetry is generated, it must be redistributed from the dark sector to the visible sector. The mechanism which transfers the asymmetry to the SM must necessarily break either baryon or lepton number, and can be either perturbative or nonperturbative. We find the most stringent constraints, perhaps not surprisingly, arise from the transfer mechanisms.

Finally, the hidden sector must satisfy some additional constraints in order to yield a satisfactory cosmology. In particular, the symmetric portion of the DM abundance must annihilate efficiently away [10], and the contribution of hidden sector states to the expansion of the Universe must be minimal by the time of nucleosynthesis; both of these conditions can be satisfied by including additional baryon, lepton, and dark number-preserving couplings between the hidden sector and the SM.

We show in Fig. 1 a schematic of the classes of models we will consider in this paper. We begin in Sec. II by building the minimal hidden sector which accomplishes darkogenesis. As the dark sector is relatively unconstrained, most of the constraints reside in the mechanism chosen for transferring the asymmetry between sectors, which we discuss in Sec. III. In Sec. IV we build two explicit models based on the minimal hidden sector, using both perturbative and nonperturbative mechanisms for transferring the asymmetry between sectors. In Sec. V, we conclude.

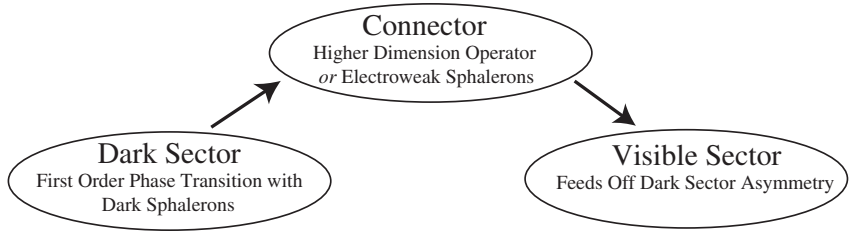

FIG. 1. A schematic of the classes of models we consider. The asymmetry is generated in the hidden sector via a first-order phase transition and then transferred to the visible sector either via a higher-dimension operator or electroweak sphalerons.

\section{A FIRST-ORDER DARK PHASE TRANSITION}

Models where the matter-antimatter asymmetry are generated from a first-order phase transition are an elegant approach to generating the observed matter abundance. The SM famously includes in principle all of the ingredients necessary for a baryon asymmetry to be generated at the electroweak phase transition, but quantitatively fails to generate the observed baryon excess. Much work has been performed on extending the matter content of the SM to increase both the magnitude of the $C P$ violation and the departure from equilibrium in order to rescue electroweak baryogenesis. Some of these models also include a DM candidate, whether thermal, as in the nearly minimal supersymmetric standard model [(n)MSSM] [11], or nonthermal, as in [12,13].

We choose to focus on the less-studied scenario where the dark sector itself has a first-order phase transition in which the SM does not directly participate. In this scenario, the necessary departure from equilibrium occurs due to the passage of supercritical bubbles of the broken phase through the plasma. In order to generate a matterantimatter asymmetry, the other two Sakharov conditions must additionally be satisfied. These can be satisfied naturally within chiral non-Abelian hidden sectors.

First, there must be a dark number-violating process which is efficient in the symmetric phase and suppressed in the broken phase, and which moreover shuts off faster than the time scale for the passage of the bubble wall. The requirement for the dark number-violating process to shut off precisely at the phase transition is nontrivial. The most natural candidate process is dark sphalerons, the rate for which becomes exponentially suppressed precisely at the phase transition. Thus we achieve dark baryogenesis via the symmetry-breaking phase transition of a dark nonAbelian gauge group, $S U(\chi)$, and require that the dark sector contain matter fields which have a global symmetry $U(1)_{D}$ which is anomalous under $S U(\chi)$.

To summarize our scenario, we have the following:

(i) If the dark sector matter fields are chiral under the dark gauge group $S U(\chi)$, this can give rise to an anomalous dark number symmetry $U(1)_{D}$ under the $S U(\chi)$. Dark number violation is then achieved by dark sphalerons.

(ii) The group $S U(\chi)$ undergoes a symmetry-breaking first-order phase transition, during which time a dark matter number asymmetry is generated through the $C$ - and $C P$-violating interactions of the dark sector matter fields with the bubble walls.

(iii) The asymmetry generated during the phase transition is transferred to the SM through one of the mechanisms outlined in Sec. III.

In our scenario $C$ violation in the dark sector is implemented through the requirement that the global dark number $U(1)_{D}$ has a chiral anomaly under the dark gauge group. 
TABLE I. Matter content in the minimal hidden sector which generates a matter-antimatter asymmetry from a first-order phase transition.

\begin{tabular}{lcc}
\hline \hline & $S U(2)_{D}$ & $U(1)_{D}$ \\
\hline$H, H^{c}$ & 2 & 0 \\
$L_{D} \times 2$ & 2 & 1 \\
$\bar{X}_{1,2} \times 2$ & 1 & -1 \\
\hline \hline
\end{tabular}

We will take the hidden sector to be weakly coupled for simplicity. Then the breaking of $S U(\chi)$ is accomplished through a fundamental scalar Higgs boson, and the interactions of the dark sector fields with the bubble wall take the form of chiral, $C P$-violating couplings to the dark Higgs. The minimal field content in the dark sector which can satisfy all conditions consists of $2 m$ fermionic doublets of an $S U(2)$ gauge group, which have an anomalous number symmetry $U(1)_{D} ; 2 \times 2 m$ fermionic singlets to form Dirac fermions after $S U(2)$-breaking; and $k$ Higgs doublet scalars. The number of fermionic $S U(2)$ doublets must be even to cancel the global anomaly. In order to yield a physical $C P$-violating phase, we must have either $m>1$ or $k>1$, so the minimal matter content consists of a two Higgs doublet model together with 2 fermionic doublets and 4 fermionic singlets. Majorana mass terms $M_{2} L_{D} L_{D}$ for doublets and $M_{1} \bar{X} \bar{X}$ for singlets are forbidden by the global dark number $U(1)_{D}$. This minimal hidden sector is summarized in Table I.

Since we are utilizing a fundamental scalar, it is appealing to embed these models within supersymmetry (SUSY) so as to make this hidden sector (as well as the visible sector) technically natural. The simplest dark supersymmetric sector which is chiral under $S U(2)_{D}$, nonanomalous, and gives masses to all states in the dark sector is obtained simply by supersymmetrizing the minimal hidden sector of Table I, and is described by the superpotential

$$
W=\mu_{D} H H^{c}+y_{1 i} L_{D} H \bar{X}_{i}+y_{2 j} L_{D} H^{c} \bar{X}_{j},
$$

where $i, j=1,2$; we suppress generational indices in Eq. (3).

This minimal dark sector is the simplest (supersymmetric) hidden sector which realizes departure from equilibrium via a first-order phase transition and satisfies all of Sakharov's criteria. It will serve as the basis for our models in Sec. IV. It is straightforward to ensure that the phase transition is sufficiently first-order given the unconstrained dynamics in the hidden sector, as we will see below. The observable phenomenology of models built on this hidden sector is dominated by the communication of the generated dark number to the visible sector, which we turn to next.

\section{ASYMMETRY COMMUNICATION MECHANISMS}

Once an asymmetry is generated in the hidden sector, it must be transferred to the SM. This may be accomplished either perturbatively or nonperturbatively. We enumerate the possibilities and comment on these transfer mechanisms, as well as on the constraints which each transfer mechanism imposes on the phase transition in the hidden sector.

\section{A. Higher dimension operators}

In order to successfully transfer a dark number asymmetry to a baryon number asymmetry, the dark sector and the visible sector must be linked by some mediator states which carry both hidden sector and SM (gauge and/or global) charges. There are many possibilities for these new degrees of freedom. Below the mass scale of these new mediators, however, the dark-visible interactions which they induce can be described using higher dimension operators in the effective theory below the mediator mass scale $\Lambda$. These contact interactions will generically remain in equilibrium for a range of temperatures below the mediator mass scale, allowing us to describe a broad range of possible dark-visible interactions in terms of a small set of higher dimensional operators which carry nonzero $B$ or $L$ as well as nonzero dark number $D$. The operator in the SM sector must also be sterile, since the DM itself is sterile. The lowest-order such operators are

$$
\begin{aligned}
\mathcal{O}_{d+5 / 2} & =\frac{\mathcal{O}_{d} L H}{\Lambda^{d-3 / 2}}, \\
\mathcal{O}_{d+9 / 2, B} & =\frac{\mathcal{O}_{d} u^{c} d^{c} d^{c}}{\Lambda^{d+1 / 2}}, \\
\mathcal{O}_{d+9 / 2, L 1} & =\frac{\mathcal{O}_{d} L L e^{c}}{\Lambda^{d+1 / 2}}, \\
\mathcal{O}_{d+9 / 2, L 2} & =\frac{\mathcal{O}_{d} L Q d^{c}}{\Lambda^{d+1 / 2}}, \\
\mathcal{O}_{d+5} & =\frac{\mathcal{O}_{d} L H L H}{\Lambda^{d+1}},
\end{aligned}
$$

where $d$ is the dimension of the dark sector operator, $\mathcal{O}_{d}$. The operator $\mathcal{O}_{d+5 / 2}$ is a special case, and if $\mathcal{O}_{d}$ corresponds to a single (fermionic) state in the dark sector, it can contribute directly to the neutrino mass matrix after electroweak symmetry breaking; such contributions are highly constrained.

In order to successfully transmit a matter-antimatter asymmetry from a hidden sector to the SM using one of the operators of Eq. (5), the hidden sector phase transition must happen above the temperature $T_{f}$ at which the operator freezes out. Moreover, if the hidden sector couples to the visible sector only through lepton number-violating operators, then the hidden sector phase transition must occur above the electroweak phase transition, so that an initial lepton asymmetry can be reprocessed into a baryon asymmetry. If, however, the coupling between the sectors proceeds through the baryon-number violating operator $\mathcal{O}_{d+9 / 2, B}$, then the phase transition can occur at lower 
scales. We will construct a low-scale darkogenesis model using this operator in Sec. IVA below.

In a supersymmetric theory, the operators of Eq. (5) can be understood as contributions to the superpotential,

$$
\begin{aligned}
\mathcal{O}_{d+2} & =\frac{\mathcal{O}_{d} L H}{\Lambda^{d-1}}, \\
\mathcal{O}_{d+3, B} & =\frac{\mathcal{O}_{d} u^{c} d^{c} d^{c}}{\Lambda^{d}}, \\
\mathcal{O}_{d+3, L 1} & =\frac{\mathcal{O}_{d} L L e^{c}}{\Lambda^{d}}, \\
\mathcal{O}_{d+3, L 2} & =\frac{\mathcal{O}_{d} L Q d^{c}}{\Lambda^{d}}, \\
\mathcal{O}_{d+4} & =\frac{\mathcal{O}_{d} L H L H}{\Lambda^{d+1}} .
\end{aligned}
$$

In supersymmetric theories it can be easier to satisfy observational constraints on these operators, as rates for baryon- or lepton-number changing processes can receive additional suppression from superpartner mass scales.

\section{B. Electroweak sphalerons}

Baryon and lepton number are also broken nonperturbatively in the SM by electroweak sphalerons. To successfully transmit a matter-antimatter symmetry from a hidden sector to the SM via electroweak sphalerons requires a chiral mediator sector: particles which carry both $S U(2)_{L}$ and the dark global symmetry, such that the dark number symmetry becomes anomalous under $S U(2)_{L}$. This enables electroweak sphalerons to reprocess a generated dark asymmetry into a SM baryon (and lepton) asymmetry. We will present a model with a simple messenger sector in Sec. IV B below.

In this scenario, the dark phase transition must again happen at temperatures above the electroweak phase transition. Moreover, the messenger fields must now obtain all of their mass from electroweak symmetry breaking, and therefore cannot be decoupled from the electroweak scale. Constraints on the messenger fields are therefore more stringent than for perturbative mediation. In particular, precision electroweak constraints on additional heavy electroweak matter as well as collider limits on direct production must be avoided.

\section{MODELS OF DARK BARYOGENESIS}

We now present two explicit models of darkogenesis based on the minimal supersymmetric dark hidden sector described in Sec. II: first, a low-scale model based on perturbative mediation through the baryon-violating operator $\mathcal{O}_{d+3, B}$ and, second, a higher-scale model which uses electroweak sphalerons to transfer the asymmetry. The common ingredient in both models is the supersymmetric version of the minimal dark hidden sector described in Sec. II, though the mass scale of the dark symmetrybreaking phase transition and therefore of the dark
Higgses $H, H^{c}$ differs between the two models. In the models presented below, we incorporate a mechanism which generates the mass scale of the dark Higgses dynamically, via a singlet which communicates to the common origin of visible and hidden sector SUSYbreaking [14,15].

Successful darkogenesis requires not only that a dark asymmetry be generated and transferred, but also that the symmetric portion of the DM abundance annihilate away. We construct the Higgs potential in the hidden sector to yield a spectrum which allows for efficient annihilation of the symmetric DM abundance as well as a first-order phase transition.

\section{A. Low-scale dark baryogenesis}

In darkogenesis models where the dark phase transition occurs at temperatures below the electroweak phase transition, the asymmetry must be transferred directly to the baryons. The lowest-dimension neutral operator which can accomplish this is $\frac{1}{\Lambda^{p}} \mathcal{O}_{d} u^{c} d^{c} d^{c}$. This operator must be in thermal equilibrium at temperatures of order the dark phase transition, but must leave equilibrium at temperatures $T_{\mathrm{dec}}$ above where the DM becomes nonrelativistic; otherwise the transfer operator will wash out the dark asymmetry. It is easy to arrange this separation of scales $T_{\mathrm{dec}}>m_{\mathrm{DM}}$ in a supersymmetric model, as any darkvisible interaction arising from the superpotential term $W_{\text {int }}=\frac{1}{\Lambda^{d}} \mathcal{O}_{d} u^{c} d^{c} d^{c}$ must involve at least one squark. This gives rise to a Boltzmann suppression in the rate for the operator, which causes it to decouple rapidly below the superpartner mass scale. Thus while there is no need for $B$ violation through rapid electroweak sphalerons in this model, the typical scales for the dark phase transition are still naturally related to the electroweak scale, through the mass scales for SM superpartners.

To build a low-scale model, we connect the SM to the supersymmetric minimal hidden sector of Sec. II using the operator

$$
W_{\text {int }}=\frac{1}{\Lambda^{2}} X^{2} u^{c} d^{c} d^{c},
$$

where $X$ is the dark matter state. We take this operator to be quadratic rather than linear in $X$ to avoid inducing $X$ decay. This interaction can be generated by integrating out (for instance) a vector pair of color triplet superfields $\zeta, \bar{\zeta}$ and a pair of singlet superfields $N, \bar{N}$, with the renormalizable interactions

$$
W=m_{\zeta} \zeta \bar{\zeta}+m_{N} N \bar{N}+d^{c} d^{c} \zeta+\bar{\zeta} u^{c} N+\bar{N} X X .
$$

Again, $U(1)_{D}$-breaking mass terms for the $U(1)_{D}$-charged singlets $N, \bar{N}$ must be forbidden. If one of the squarks is light, $m_{\tilde{q}} \sim 200 \mathrm{GeV}$, the decoupling temperature for this operator can be quite low: for DM masses $m_{\tilde{X}} \sim m_{X}=$ $10 \mathrm{GeV}$ and taking $\Lambda \sim \mathrm{TeV}, \tilde{X} \tilde{X} \rightarrow q q \tilde{q}$ drops below the expansion rate of the Universe at $T \sim 50 \mathrm{GeV}$. 
The relation between the baryon number asymmetry $B$ and the dark number asymmetry $D$ can be determined using the standard methods outlined in [16]. If the transfer operator freezes out after electroweak sphalerons have decoupled, we find

$$
\frac{B}{D}=\frac{23}{21},
$$

taking for concreteness one (Dirac) DM state and its superpartners in the thermal plasma. The DM mass is then determined to be

$$
m_{X}=5 \frac{B}{D} m_{p} \approx 5 \mathrm{GeV} .
$$

Out-of-equilibrium decays of the SM next-to-lightest supersymmetric particle (NLSP) through the transfer operator could potentially alter this relation, but in our model do not, as we will see below. In the minimal weakly coupled hidden sector the DM mass is controlled by a technically natural Yukawa coupling and can be freely adjusted. However, as the masses of all light states in the hidden sector are parametrically given by their couplings times the scale of dark symmetry breaking, once the scale of the phase transition and the mass of the dark matter have been specified the strength of the hidden sector interactions are no longer adjustable.

In order to show that the Sakharov criteria can be satisfied via our superpotential, Eq. (3), we must examine some of the details of the phase transition in the hidden sector. Rather than setting the mass scale $\mu_{D}$ by hand, we generate it dynamically via singlet mediation $[14,15]$ which communicates SUSY breaking from a gaugemediated supersymmetry breaking messenger sector to the hidden sector. This mechanism also provides the means to radiatively break the dark $S U(\chi)$ in the hidden sector. We discuss this model as an example of how one could successfully carry out dark baryogenesis in the hidden sector given by Eq. (3). Other models could be constructed. We take the messenger scale to be sufficiently low that the gravitino is lighter than the lightest state in the hidden sector. We will see that the hidden Higgs potential thus generated can naturally have a first-order phase transition.

To this end, we replace the dark $\mu$ term, $\mu_{D} H H^{c}$, with the singlet terms

$$
W_{\mathrm{dh}}=\lambda S H H^{c}+\frac{\kappa}{3} S^{3} .
$$

We assume that $S$ obtains a weak scale SUSY-breaking mass, and furthermore that the soft mass-squared for the scalar is positive. It is possible to achieve this SUSYbreaking pattern via coupling to a SUSY-breaking sector if the couplings are $R$-symmetric. No bare $B / \mu$ is generated, and $A$ terms in the potential $\lambda A_{\lambda} S H H^{c}$ and $\kappa A_{\kappa} S^{3}$ only arise through renormalization group flow. Negative soft mass-squareds are then obtained for the dark Higgses $H, H^{c}$ through one-loop diagrams:

$$
m_{H}^{2}=m_{H^{c}}^{2} \simeq-\frac{2 \lambda^{2}}{16 \pi^{2}} m_{S}^{2} \ln \left(\frac{\Lambda}{m_{\mathrm{hid}}}\right),
$$

where $m_{\text {hid }}$ is the mass scale of the hidden sector, and $\Lambda$ is the scale where the singlet mass is generated.

Without the presence of an additional dark quantum number, the two Higgs doublets of the hidden sector are indistinguishable. In that case the general soft terms will then contain arbitrary mixings between the doublets, $\mathcal{L}_{\text {dh, gen }}=m_{i j}^{2} \bar{H}_{i} H_{j}$, where $H_{i}, H_{j}=H, H^{c}$. These more general Higgs quadratics complicate the minimization of the potential. To simplify the discussion we impose a global symmetry on the hidden Higgs potential so that it reduces to an (N)MSSM-like form. There is, however, no problem in principle with allowing all terms mixing the Higgses. Imposing the symmetry poses no cosmological problems, as it is explicitly broken by the Yukawa couplings, preventing the formation of domain walls. As in the NMSSM, with this potential only one component of each of dark Higgs (call it "0") will obtain a vacuum expectation value (VEV), and these VEVs are located in opposite isospin components. The potential for these degrees of freedom can be written

$$
\begin{aligned}
V= & \lambda^{2}|S|^{2}\left(\left|H_{0}^{c}\right|^{2}+\left|H_{0}\right|^{2}\right)+\lambda^{2}\left|H_{0}^{c} H_{0}\right|^{2} \\
& +\frac{g_{D}^{2}}{8}\left(\left|H_{0}\right|^{2}-\left|H_{0}^{c}\right|^{2}\right)^{2}+m_{H}^{2}\left|H_{0}\right|^{2}+m_{H_{0}^{c}}^{2}\left|H^{c}\right|^{2} \\
& +m_{S}^{2}|S|^{2}+\kappa|S|^{4}+\left(-\lambda \kappa H_{0}^{c} H_{0} \bar{S}^{2}-\lambda A_{\lambda} S H_{0}^{c} H_{0}\right. \\
& \left.+\frac{\kappa}{3} S^{3}+\text { H.c. }\right) .
\end{aligned}
$$

The symmetry-breaking pattern is

$$
\left\langle H_{0}\right\rangle^{2} \simeq\left\langle H_{0}^{c}\right\rangle^{2} \equiv \eta^{2} \simeq-\frac{m_{H}^{2}}{\lambda^{2}}
$$

and

$$
\langle S\rangle \equiv s=\frac{\lambda A_{\lambda} \eta^{2}}{m_{S}^{2}} .
$$

The minimum is stable provided $g_{D}^{2}-2 \lambda^{2}>0$.

We now discuss the spectrum of the hidden sector in more detail to ensure that there are no cosmological issues. There are five physical Higgses associated with $H, H^{c}$ and two Higgses associated with $S$. The real component of $S$ remains heavy, $m_{h_{s}}^{2} \simeq m_{S}^{2}$. There are three nearly degenerate Higgses (corresponding to one "neutral" Higgs and two "charged" Higgses) with masses $m_{h_{1}}^{2} \simeq$ $\left(g_{D}^{2}-2 \lambda^{2}\right) \eta^{2}$, and one lighter Higgs with mass $m_{h_{2}}^{2} \simeq$ $2 \lambda^{2} \eta^{2}$. In the pseudoscalar sector, the theory has a global symmetry in the limit $\kappa \rightarrow 0$ or $A_{\lambda, \kappa} \rightarrow 0$, which is spontaneously broken by $\eta$, so that there is a Goldstone boson. The pseudoscalar masses are the mostly singlet $m_{a_{s}}^{2} \simeq m_{S}^{2}$ 
and the mostly doublet $m_{a_{h}}^{2} \simeq 6 \frac{s^{2}}{\eta^{2}}\left(-3 \lambda \kappa \eta^{2}+\kappa A_{\kappa} s\right)$ (see for example $[15,17]$ for details).

The neutralino mass matrix, in the limit $s \ll \eta$, is [in the $\left(\tilde{\lambda}, \tilde{H}, \tilde{H}^{c}, \tilde{S}\right)$ basis $]$

$$
\mathcal{M}^{f}=\frac{1}{\sqrt{2}}\left(\begin{array}{cccc}
0 & g_{D} \eta & -g_{D} \eta & 0 \\
g_{D} \eta & 0 & 0 & \sqrt{2} \lambda \eta \\
-g_{D} \eta & 0 & 0 & \sqrt{2} \lambda \eta \\
0 & \sqrt{2} \lambda \eta & \sqrt{2} \lambda \eta & 0
\end{array}\right),
$$

giving two fermions with mass $M_{1,2}^{0}=g_{D} \eta$ and two fermions with mass $M_{3,4}^{0}=\sqrt{2} \lambda \eta$. These are nearly degenerate with the Higgses $m_{h_{1}}$ and $m_{h_{2}}$. In addition, the charginos have masses $M^{ \pm}=g_{D} \eta$. A sample spectrum is shown in Fig. 2.

The DM candidate is the lightest of the states carrying $U(1)_{D}$, which we will henceforth denote $X$. The mass of the DM state is largely controlled by the Yukawa couplings in the superpotential of Eq. (3), with mass splittings within the chiral multiplets subdominant. Since $H, H^{c}$ receive negative soft mass-squareds, within each matter multiplet the scalar will be heavier than the fermion. This implies that the DM $X$ is fermionic, while its superpartner $\tilde{X}$ will decay to $X$ and the gravitino on a time scale

$$
\begin{aligned}
\tau(\tilde{X} \rightarrow X \tilde{G}) \simeq & \frac{16 \pi\langle F\rangle^{2} m_{X}^{3}}{\Delta m_{X}^{8}} \\
\simeq & \left(3 \times 10^{4} \mathrm{~s}\right)\left(\frac{\sqrt{F}}{100 \mathrm{TeV}}\right)^{4}\left(\frac{m_{X}}{10 \mathrm{GeV}}\right)^{3} \\
& \times\left(\frac{10^{-3}(10 \mathrm{GeV})^{2}}{\Delta m_{X}^{2}}\right)^{4},
\end{aligned}
$$

where $\Delta m_{X}^{2} \ll m_{X}^{2}$ is the mass-squared splitting between the scalar and fermion induced by radiative corrections,

$$
\Delta m_{X}^{2}=-\frac{2 y^{2}}{16 \pi^{2}} m_{H}^{2} \log \left(\frac{M_{\mathrm{mess}}}{m_{X}}\right) .
$$

This decay should have a negligible effect on cosmology, however, because both $X$ and $\tilde{X}$ have efficient annihilations to the light pseudoscalar $a_{h}$.

The light pseudoscalar provides an efficient annihilation mechanism for removing the symmetric abundance of these particles. Let $y$ denote the effective Yukawa coupling of the DM state to the light pseudoscalar, $-\mathcal{L}_{\text {int }} \equiv$

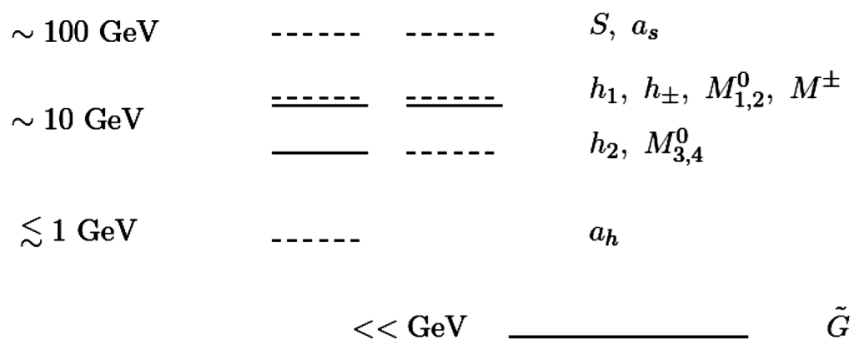

FIG. 2. The spectrum of the minimal dark sector. Among the states carrying $U(1)_{D}$, only the lightest, $X$ (the DM particle), is shown. $i y a_{h} \bar{X} \gamma^{5} X$. Then the annihilation cross section for the process $\tilde{X}^{*} \tilde{X} \rightarrow a_{h} a_{h}$ is

$\langle\sigma v\rangle \simeq \frac{y^{4}}{64 \pi} \frac{1}{m_{X}^{2}} \simeq\left(2 \times 10^{-24} \mathrm{~cm}^{3} / \mathrm{s}\right)\left(\frac{y}{0.25}\right)^{4}\left(\frac{10 \mathrm{GeV}}{m_{X}}\right)^{2}$,

which is sufficiently large to efficiently remove the symmetric component.

The axion itself will be stable in the absence of any interactions with the SM. Since it is massive, and its evolution thermal, its abundance could be problematic cosmologically. On the other hand, if it has a small coupling to the SM through a term $\Delta W=\zeta S H_{u} H_{d}$, it can decay via the SM Higgs to photon pairs. The lifetime for this decay is as given in $[15,18]$

$$
\begin{aligned}
\tau \simeq & \frac{256 \pi^{3}}{\alpha^{2} \lambda^{2} \zeta^{2}} \frac{1}{F\left(m_{a_{s}}, \tan \beta\right)^{2}} m_{A}^{4} \eta^{2} m_{a_{s}}^{3} \\
\simeq & (0.003 \mathrm{~s})\left(\frac{10^{-3}}{\zeta}\right)^{2}\left(\frac{0.1}{\lambda}\right)^{2}\left(\frac{225}{F\left(m_{a_{s}}, \tan \beta\right)^{2}}\right) \\
& \times\left(\frac{m_{A}}{100 \mathrm{GeV}}\right)^{4}\left(\frac{40 \mathrm{GeV}}{\eta}\right)^{2}\left(\frac{m_{a_{s}}}{0.1 \mathrm{GeV}}\right)^{3},
\end{aligned}
$$

where $m_{A}$ is the MSSM pseudoscalar mass, $\tan \beta$ is the ratio of MSSM Higgs VEVs, and $F\left(m_{a_{s}}, \tan \beta\right)$ is obtained by summing over the contribution of SM fermions,

$$
\begin{aligned}
& F\left(m_{a_{s}}, \tan \beta\right) \\
& \quad=\sum_{i} N_{c i} Q_{i}^{2}\left(-2 \frac{4 m_{i}^{2}}{m_{a_{s}}}\right) f\left(4 m_{i}^{2} / m_{a_{s}}\right) \begin{cases}\cot \beta & \text { up-type } \\
\tan \beta & \text { down-type }\end{cases}
\end{aligned}
$$

with

$$
f(\tau)=\left\{\begin{array}{ll}
\left(\sin ^{-1}(1 / \sqrt{\tau})\right)^{2} & \tau \geq 1 \\
-\frac{1}{4}\left(\ln \frac{1+\sqrt{1+\tau}}{1+\sqrt{1+\tau}}-i \pi\right)^{2} & \tau<1
\end{array} .\right.
$$

The coupling to the visible sector through $\zeta S H_{u} H_{d}$ can be efficient enough to allow the axions to decay before they come to dominate the total energy density. We find that this is sufficient for a $0.1 \mathrm{GeV}$ axion for $\zeta \geqslant 10^{-3}$.

The small symmetric coupling through the Higgs portal also affects the decay of the SM NLSP. For $\zeta \gtrsim 10^{-3}$, if 
the SM NLSP contains any $\tilde{W}_{3}$ admixture, the decay to a hidden sector Higgs and Higgsino will dominate over both its decay to gravitino as well as potential baryon- and darknumber violating decays through the transfer operator. This ensures the symmetric abundance of both $B$ and $D$ is not repopulated; meanwhile, the hidden sector Higgsino can decay rapidly to the axion and the gravitino.

In order to understand whether a nonzero dark number asymmetry is generated during the phase transition we must consider the scalar potential at finite temperature and verify that this spectrum is consistent with a first-order phase transition. A complete calculation of the order of the phase transition would require a careful numerical study; to obtain a basic estimate of the requirements on the hidden sector couplings we perform a simplified approximate analysis. We work below the heavy singlet scale $m_{s}$, and neglect terms which are higher order in the small $S$ VEV. Moreover, we make the simplifying assumption that the ratio of hidden sector Higgs VEVs does not substantially change as a function of temperature, and consider only fluctuations in the linear combination of fields responsible for gauge symmetry breaking, $\phi=\sqrt{|H|^{2}+\left|H^{c}\right|^{2}}$. Neglecting the terms which involve the small $S$ VEV, the zero-temperature potential for this degree of freedom is then simply

$$
V=m_{H}^{2} \phi^{2}+\frac{\lambda^{2}}{4} \phi^{4},
$$

where $m_{H}^{2} \equiv m_{H_{0}}^{2}=m_{H_{0}^{c}}^{2}$. The finite temperature thermal potential is given by

$$
V(\phi, T)=V_{0}(\phi)+V_{1}(\phi, T)+\cdots,
$$

where $V_{1}(\phi, T)$ is the one-loop contribution. As $g_{D}>2 \lambda$, the leading contribution to $V_{1}(\phi, T)$ is from the (transversely polarized) gauge bosons, which to leading order give

$$
\begin{aligned}
V_{1}^{(\text {gauge })}(\phi, T) & =\frac{g_{g}}{24} m^{2}(\phi) T^{2}-\frac{g_{g}}{12 \pi}\left(m^{2}(\phi)\right)^{3 / 2} T+\cdots \\
& =\frac{1}{8} g_{D}^{2} \phi^{2} T^{2}-\frac{1}{4 \sqrt{2} \pi} g_{D}^{3} \phi^{3} T+\cdots,
\end{aligned}
$$

where $g_{g}$ counts the number of (transverse) degrees of freedom. Adding this one-loop piece (24) to the zerotemperature potential (22) gives

$$
\frac{\sqrt{2}\left\langle\phi\left(T_{c}\right)\right\rangle}{T_{c}}=\frac{g_{D}^{3}}{2 \pi \lambda^{2}} .
$$

The gauge bosons by themselves are then sufficient to drive the dark phase transition to be sufficiently strongly firstorder provided $\lambda$ is sufficiently small, $g_{D}^{3} / 2 \pi \gtrsim \lambda^{2}$.

Direct detection in this model is controlled by the small symmetric coupling through the Higgs portal. In the limit of large $\tan \beta$, the scattering cross section per nucleon is

$$
\begin{aligned}
\sigma \simeq & \frac{\mu_{r}^{2}}{\pi} N_{n}^{2} y_{h}^{2}\left(\frac{\lambda \zeta v_{u} \eta}{m_{h}^{2}}\right)^{2} \frac{1}{m_{h_{2}}^{4}} \\
\approx & 2 \times 10^{-43} \mathrm{~cm}^{2} y^{2}\left(\frac{\mu_{n}}{m_{p}}\right)^{2}\left(\frac{N_{n}}{0.1}\right)^{2}\left(\frac{\lambda}{0.1}\right)^{2}\left(\frac{\eta}{20 \mathrm{GeV}}\right)^{2} \\
& \times\left(\frac{\zeta}{10^{-3}}\right)^{2}\left(\frac{115 \mathrm{GeV}}{m_{h}}\right)^{4}\left(\frac{10 \mathrm{GeV}}{m_{h_{2}}}\right)^{4},
\end{aligned}
$$

where $\mu_{r}$ is the DM-nucleon reduced mass, $y_{h} \sim m_{D} / \eta$ is the effective Yukawa coupling of the dark matter to the light dark Higgs eigenstate, and $N_{n}$ is the coupling of the MSSM Higgs to the nucleons. This is in reach of direct detection experiments.

DM can additionally scatter off visible matter via the baryon-number violating transfer operator $W_{\text {int }}=$ $\frac{1}{\Lambda^{2}} X^{2} u^{c} d^{c} d^{c}$. In the present model, since the splitting between the DM state and its superpartner is smaller than the proton-pion mass splitting, the dominant baryon-violating scattering process is

$$
p^{+} X \rightarrow \tilde{\bar{X}} \pi^{+} .
$$

This process must proceed through a loop of SM superpartners. The rate for this process is on the order of $10^{-30} / \mathrm{yr}$ for $\mathrm{TeV}$-scale superpartners. While the present bounds on the proton lifetime are $8.2 \times 10^{33}$ years (for $p^{+} \rightarrow e^{+} \pi^{0}$ ) and $6.6 \times 10^{33} \mathrm{yr}\left(\right.$ for $p^{+} \rightarrow \mu^{+} \pi^{0}$ ) [19], these limits are not applicable to the process of Eq. (27), where the pion is the only visible particle in the final state.

\section{B. Mirror messengers and high-scale dark baryogenesis}

If the dark phase transition occurs above the electroweak phase transition, a generated dark asymmetry can be communicated to the SM via electroweak sphalerons, instead of through higher-dimension operators. This mechanism requires the introduction of messenger fields which carry both $S U(2)_{L}$ and $U(1)_{D}$ quantum numbers, such that $U(1)_{D}$ becomes anomalous under $S U(2)_{L}$. We will call these chiral messengers leptodarks, as in our model they will have leptonlike SM charges, in addition to carrying dark number.

In order to avoid fractionally charged states after electroweak symmetry breaking (EWSB), we must either ensure any fractionally charged leptodarks are bound into integrally charged composites, as in [8], or assign hypercharge to the leptodarks in such a way that the resulting states after electroweak symmetry breaking are integrally charged. This ensures that the lightest messenger can decay.

A minimal chiral messenger sector is shown in Table II. The messenger sector carries vectorlike SM quantum numbers, ensuring anomaly cancellation, and chiral $U(1)_{D}$ quantum numbers. The hypercharge assignments are necessary to ensure that all states after EWSB have integral charge. The superpotential in this messenger sector takes on the form 
TABLE II. A minimal dark messenger sector. Anomaly cancellation is achieved via mirror fermions with the same $U(1)_{D}$ charge, but opposite hypercharge. There are two sterile states, $\bar{X}_{M}^{1,2}$.

\begin{tabular}{lccc}
\hline \hline & $S U(2)_{L}$ & $U(1)_{Y}$ & $U(1)_{D}$ \\
\hline$L_{\bar{M}}^{ \pm}$ & 2 & $\pm \frac{1}{2}$ & 1 \\
$e_{M}^{c}{ }^{ \pm}$ & 1 & $\mp 1$ & -1 \\
$\bar{X}_{M}^{i}$ & 1 & 0 & -1 \\
\hline \hline
\end{tabular}

$$
\begin{aligned}
W_{M}= & y_{e M+} L_{M}^{+} H_{u} e_{M}^{c}+y_{e M-} L_{M}^{-} H_{d} e_{M}^{c}{ }^{-} \\
& +y_{X M+} L_{M}^{+} H_{d} \bar{X}_{M}^{i}+y_{X M-} L_{M}^{-} H_{u} \bar{X}_{M}^{i} .
\end{aligned}
$$

The messengers must also carry the same global quantum number as the hidden sector $U(1)_{D}$.

The messenger fields contribute to precision electroweak observables. In the limit where the mass splitting between charged and neutral leptodarks goes to zero, the messenger contribution to precision electroweak observables is

$$
\Delta S=\frac{1}{3 \pi} \simeq 0.11, \quad \Delta T \simeq \Delta U \simeq 0,
$$

which is compatible at 95\% C.L. with observations [20,21]. Agreement with data can be further improved by adjusting the mass splittings between the components of the leptodark multiplets. The most stringent collider constraints on the messengers are the LEP mass limits, $m_{L^{ \pm}}>100.8 \mathrm{GeV}$ for charged leptodarks and $m_{N}>$ $45.0 \mathrm{GeV}$ for neutral leptodarks [20,22].

The details of the dark Higgs potential can be taken to be the same as the singlet-mediated example for the low-scale model, with the overall mass scale translated to values above the weak scale to trigger the early phase transition. This can occur if the soft singlet mass is now significantly above the weak scale, a viable option depending on the couplings of the singlet to the source of SUSY-breaking. Raising the intrinsic hidden sector scale so far above the dark matter mass scale means, in our minimal sector, that the interactions of the light hidden sector degrees of freedom become weak, and generically additional structure is required to remove the thermal relic abundance. Communication between the dark and messenger sectors as the electroweak sphalerons freeze out can be ensured either by allowing a significant mixing between the messenger and dark singlets, or by introducing an additional explicit coupling $W_{\text {int }}=\xi Z \bar{X}_{D} X_{M}$ involving a singlet superfield $Z$, and where we have added a subscript to the dark singlets $\bar{X}_{D}$ for clarity. In this latter case, the $U(1)_{D}$ quantum numbers for the messenger fields are reversed from those chosen in Table II.

The DM is again fermionic, and consists of a dark singlet $\bar{X}_{D}$ paired with one component of a dark doublet $L_{D}$ with possible admixtures from the neutral component of a messenger doublet $L_{M}$ controlled by the mixing angle $\sin \chi \approx \chi$. To avoid constraints from the invisible $Z^{0}$ width, the messenger doublet component in the DM state should be small, $\chi^{4} \leq 10^{-3}$.

This model, having a high-scale phase transition, suffers from a greater weakness than the low-scale model because there are naturally no additional light states present for the dark state to annihilate efficiently to. One can remedy this by introducing additional light degrees of freedom in the hidden sector. For example, one can introduce an additional pair of matter multiplets in the hidden sector, $Y$ and $\bar{Y}$, which are $S U(2)_{D}$ singlets, carry dark number +1 and -1 respectively, and interact with the DM through

$$
W_{Y}=m_{Y} \bar{Y} Y+\xi_{Y} Z \bar{X}_{D} Y \text {. }
$$

This interaction allows the dark matter to annihilate to light $Z$ fermions with a cross section

$$
\begin{aligned}
\langle\sigma v\rangle(X \bar{X} \rightarrow \tilde{z} \tilde{z}) & =\frac{\xi_{Y}^{4}}{32 \pi m_{X}^{2}}\left(\frac{m_{X}}{m_{Y}}\right)^{4} \\
& =2 \times 10^{-24} \mathrm{~cm}^{3} / \mathrm{s} \xi_{Y}^{4}\left(\frac{m_{X}}{\mathrm{GeV}}\right)^{2}\left(\frac{15 \mathrm{GeV}}{m_{Y}}\right)^{4} .
\end{aligned}
$$

The scalars $\tilde{X}$ can decay to $X \tilde{G}$ before the $X$ annihilation process freezes out. The $Z$ scalar similarly decays to the massless fermionic $\tilde{z}$, which is stable. As long as the $\tilde{z}$ fermions are sufficiently light, this causes no problem with constraints on additional degrees of freedom from big bang nucleosynthesis (BBN), since the hidden sector is much cooler than the SM, having decoupled from the visible sector before the QCD phase transition.

With the introduction of the messenger $S U(2)_{L}$ doublets, the electroweak sphalerons now violate the global $U(1)$ number $B+L+\frac{N_{D}}{N_{g}} D$, where $N_{g}=3$ is the number of SM generations and $N_{D}=2$ is the number of messenger electroweak doublets. This reprocesses the DM number asymmetry generated from the dark phase transition into SM $B$ and $L$.

The precise relation between the baryon asymmetry and the dark asymmetry depends on a number of factors, such as how rapidly the sphalerons decouple during the electroweak phase transition, whether the top quark is integrated out of the theory when the sphalerons decouple, and how many dark and messenger fields have their mass below the electroweak phase transition. For concreteness, we take the top quark as well as all of the messengers to be heavier than the sphaleron decoupling temperature, and we take only the DM state and its superpartner integrated into the theory below the electroweak phase transition. All other hidden sector states we integrate out. In this case, assuming the electroweak phase transition is second-order, we find the dark asymmetry is related to the baryon asymmetry as

$$
\frac{B}{D}=\frac{33}{127}
$$

predicting a DM mass of approximately $1 \mathrm{GeV}$. 
With the introduction of the messenger electroweak doublets, direct detection can now proceed via the doublet fraction of the DM state as well as through mixing between the hidden and visible Higgses. The DM-neutron cross section from the doublet component of the DM state scattering through the $Z^{0}$ is

$$
\sigma=\frac{G_{F}^{2} \chi^{4} \mu_{r}^{2}}{512 \pi} \approx 5 \times 10^{-42} \mathrm{~cm}^{2}\left(\frac{\mu_{r}}{m_{p}}\right)^{2}\left(\frac{\chi}{0.1}\right)^{4},
$$

where $\chi$ is again the effective coupling of the DM to the $Z^{0}$ via mixing with the messenger doublets. This is large enough to be constrained by monojet searches at the Tevatron [23].

\section{SUMMARY AND GENERAL COMMENTS ABOUT DARKOGENESIS}

Our purpose in this paper was to outline the general requirements of a dark sector which can accomplish dark baryogenesis via a first-order phase transition in the hidden sector. We constructed a minimal weakly coupled dark sector which generates a matter-antimatter asymmetry, and discussed messenger sectors for transferring the asymmetry to the SM. We focused on a scenario where dark number violation and out-of-equilibrium dynamics in the hidden sector can be achieved via a dark non-Abelian gauge group with a first-order phase transition, under which dark number is anomalous. The chiral anomaly of the dark number current provides $C$ violation, and $C$ and $C P$ are both violated through chiral couplings of dark states to the dark Higgses. In these models the DM mass lies in a low mass window, between approximately 1 and $15 \mathrm{GeV}$, and the low symmetry-breaking scale for the dark sector can be generated dynamically via the mediation of SUSY breaking. We constructed an explicit dark Higgs sector which satisfies the requirements for a strong firstorder phase transition, and in addition provides a mechanism for successful removal of all nonasymmetric relics in the dark sector. The minimal weakly coupled hidden sector which is the basis of our models has the special feature that the interaction strengths of all light states are determined by the ratio of their mass to the symmetry-breaking scale in the hidden sector. As the dark matter must be sufficiently strongly interacting to remove its thermal relic abundance, this hidden sector requires low scales for the dark phase transition or additional structure in the dark sector. We showed that when this phase transition occurs below the electroweak phase transition (so that baryon number violation through electroweak sphalerons is off), that the dark asymmetry can still be transferred efficiently to the baryons via a higher-dimension operator. Alternatively, the dark phase transition can occur well before the electroweak phase transition. In this case, a messenger sector which carries both $S U(2)_{L}$ and the dark number can render dark number anomalous under $S U(2)_{L}$, thereby transferring the dark asymmetry into baryons. In both scenarios, the transfer mechanism is $C P$-conserving, and contributions to standard model $C P$-violating observables are suppressed.

Direct detection cross sections in these models depend on small symmetric connections between the hidden sector and the visible sector, which have no intrinsic connection to the darkogenesis mechanism or the relation between the DM and baryon number. The gravitational wave signal from the first-order phase transition could provide an orthogonal probe of our darkogenesis scenario, and for transition temperatures in the interesting range from below $100 \mathrm{GeV}$ to $100 \mathrm{TeV}$ is potentially within reach at upcoming gravitational wave observatories [24].

Many other interesting and viable scenarios of darkogenesis remain to be developed. For example, $C P$ violation could be introduced through the coupling of the dark states to the visible sector, rather than of the non-Abelian hidden sector to itself. With the great freedom offered by nontrivial dark sectors, many further novel avenues remain to be explored.

\section{ACKNOWLEDGMENTS}

We thank R. Gran for comments about SuperKamiokande constraints, and M. Buckley, H. Davoudiasl, R. Harnik, G. Kribs, D. Morrissey, L. Randall, J. Thaler, W. Skiba, and T. Volansky for useful comments and discussions. We thank the Aspen Center for Physics (J.S. and K. M.Z.) and SLAC (J.S.) for hospitality during the final stages of this work. J. S. was supported in part by DOE Grant No. DE-FG02-92ER40704.
[1] M. S. Carena, M. Quiros, and C. E. M. Wagner, Phys. Lett. B 380, 81 (1996).

[2] M.S. Carena, M. Quiros, and C.E. M. Wagner, Nucl. Phys. B524, 3 (1998).

[3] G. B. Gelmini, L. J. Hall, and M. J. Lin, Nucl. Phys. B281, 726 (1987); S. M. Barr, Phys. Rev. D 44, 3062 (1991); S. Dodelson, B. R. Greene, and L. M. Widrow, Nucl. Phys. B372, 467 (1992); M. Fujii and T. Yanagida, Phys. Lett. B
542, 80 (2002); D. Hooper, J. March-Russell, and S. M. West, Phys. Lett. B 605, 228 (2005); R. Kitano and I. Low, Phys. Rev. D 71, 023510 (2005); M. Y. Khlopov and C. Kouvaris, Phys. Rev. D 77, 065002 (2008); R. Kitano, H. Murayama, and M. Ratz, Phys. Lett. B 669, 145 (2008); G. R. Farrar and G. Zaharijas, Phys. Rev. Lett. 96, 041302 (2006); Z. Berezhiani and A. Lepidi, Phys. Lett. B 681, 276 (2009); Y. Cai, M. A. Luty, and D.E. Kaplan, 
arXiv:0909.5499; H. An, S.-L. Chen, R. N. Mohapatra et al., J. High Energy Phys. 03 (2010) 124.

[4] D. B. Kaplan, Phys. Rev. Lett. 68, 741 (1992).

[5] D. E. Kaplan, M. A. Luty, and K. M. Zurek, Phys. Rev. D 79, 115016 (2009).

[6] S. Nussinov, Phys. Lett. 165B, 55 (1985); S. M. Barr, R. S. Chivukula, and E. Farhi, Phys. Lett. B 241, 387 (1990); S. B. Gudnason, C. Kouvaris, and F. Sannino, Phys. Rev. D 73, 115003 (2006).

[7] T. Cohen and K. M. Zurek, Phys. Rev. Lett. 104, 101301 (2010).

[8] G. D. Kribs, T. S. Roy, J. Terning, and K. M. Zurek, Phys. Rev. D 81, 095001 (2010).

[9] A.D. Sakharov, Pis'ma Zh. Eksp. Teor. Fiz. 5, 32 (1967) [JETP Lett. 5, 24 (1967)].

[10] T. Cohen, D. J. Phalen, A. Pierce et al., Phys. Rev. D 82, 056001 (2010).

[11] A. Menon, D.E. Morrissey, and C.E. M. Wagner, Phys. Rev. D 70, 035005 (2004).

[12] S. Das, P. J. Fox, A. Kumar, and N. Weiner, arXiv:0910.1262.

[13] N. Craig and J. March-Russell, arXiv:1007.0019.

[14] K. M. Zurek, Phys. Rev. D 79, 115002 (2009).
[15] D. E. Morrissey, D. Poland, and K. M. Zurek, J. High Energy Phys. 07 (2009) 050.

[16] J. A. Harvey and M. S. Turner, Phys. Rev. D 42, 3344 (1990).

[17] B. A. Dobrescu and K. T. Matchev, J. High Energy Phys. 09 (2000) 031.

[18] J. F. Gunion, G. Gamberini, and S. F. Novaes, Phys. Rev. D 38, 3481 (1988).

[19] H. Nishino et al. (Super-Kamiokande Collaboration), Phys. Rev. Lett. 102, 141801 (2009).

[20] G. D. Kribs, T. Plehn, M. Spannowsky et al., Phys. Rev. D 76, 075016 (2007).

[21] See LEP EWWG, Preliminary Constraints on the Standard Model at http://lepewwg.web.cern.ch/LEPEWWG/plots/ summer2006 (s06_stu_contours.eps).

[22] C. Amsler et al. (Particle Data Group), Phys. Lett. B 667, 1 (2008) and 2009 partial update for the 2010 edition.

[23] J. Goodman, M. Ibe, A. Rajaraman et al., arXiv:1005.1286; Y. Bai, P. J. Fox, and R. Harnik, arXiv:1005.3797.

[24] A. Kosowsky, M. S. Turner, and R. Watkins, Phys. Rev. Lett. 69, 2026 (1992); C. Grojean and G. Servant, Phys. Rev. D 75, 043507 (2007). 